\title{
Value of computed tomography for selecting patients with bullous lung disease for surgery
}

\author{
M D L MORGAN, D M DENISON, B STRICKLAND \\ From the Departments of Lung Function and of Radiology, Brompton Hospital, London
}

ABSTRACT Computed tomography and lung function tests were performed on 43 patients who had evidence on the chest radiograph suggesting bullous emphysema. After computed tomography scan two groups of patients could be identified. Twenty patients had generalised emphysema, which was locally worse in the area of the suspected bulla; and 23 had well defined bullae, which were potentially operable. Results of lung function tests did not distinguish between the two groups. The volume and ventilation of the true bullae were measured by computed tomography and this confirmed that most of them did not contribute to ventilation (residual volume (RV)/total capacity (TLC) bulla $=89 \%$ (SD 10\%). The patients with true bullae were considered suitable for surgery but only 12 had an operation. All the patients who underwent surgery survived and had a symptomatic improvement, which was accompanied by objective increases in spirometric volumes and by reductions in static lung volumes; there were no improvements in carbon monoxide transfer or blood gas tensions. It is concluded that computed tomography used alone can identify bullae that are amen-able to surgery and can measure their volume and ventilation. The surgical removal of such clearly identified bullae is safe and associated with symptomatic and functional improvement even when the preoperative $\mathrm{FEV}_{1}$ is less than 1 litre. This improvement is likely to be a consequence of reduction in lung volume and may not necessarily be associated with relief of compressed peribullous lung or the removal of dead space.

When patients present with large bullae in association with chronic obstructive lung disease it may be difficult to decide whether to remove the bulla. It is important to assess these cases correctly because on occasion severely disabled patients will improve dramatically after operation. Recently, Pearson and Ogilvie have shown that this improvement can be maintained and also that surgery does not predispose to formation of further bullae. ${ }^{1}$ Recommending surgery for a patient with a large symptomatic bulla and well preserved lung function is seldom difficult. Current investigations, however, are insufficiently sensitive to identify which patients with poor overall lung function will improve after surgery to remove a relatively small bulla. ${ }^{23}$ This is believed to be especially true if the $\mathrm{FEV}_{1}$ is below 1 litre. Computed tomography is now acknowledged to be superior to conventional radiology in the identification of bullae

Address for correspondence: Dr MDL Morgan, Department of Chest Medicine, East Birmingham Hospital, Birmingham B9 5ST.

Reprints will not be available.

Accepted 23 June 1986 and could be used to measure lung volume and ventilation in such cases. ${ }^{4-6}$ This paper reports the value of computed tomography used as the primary investigation to select patients with poor lung function for surgery and also relates the size of the bullae to the postoperative change in lung function.

\section{Patients and methods}

Over a period of three years 43 patients were referred for advice because their chest radiographs showed local areas of translucency, and the referring physician considered that surgical removal of an area of bullous lung disease might benefit the patient. The plain films were then reviewed by an experienced radiologist. A bulla was defined as an area of transradiancy not containing blood vessels, which may be confined by visible bulla walls. These walls could be either continuous curves in a solitary bulla or a mixture of arcs and linear densities in multiple bullae. After the suspicion of bullous disease had been confirmed on the plain radiographs every patient underwent computed tomography in inspiration and 
expiration, and had full routine lung function tests. Most patients also had blood gas estimations. After computed tomography 23 patients with localised discrete bullae were distinguished from the remainder, who had disease that was considered to be inoperable. The patients with true bullae were recommended for surgery and had repeat lung function tests if they had an operation.

\section{COMPUTED TOMOGRAPHY}

Computed tomography was used to define the extent of the disease and also to examine the quality of nonbullous lung. All scans were performed in the same manner. In the early part of the study they were taken on a machine with a 5.3 second scan time and an image matrix of $256 \times 256$ pixels (Elscint 905). Since the beginning of 1984 we have used a more modern scanner with the same scan time but a greater image resolution-namely, $512 \times 512$ pixels (Elscint 2002). In all cases the protocol was the same. The patient lay supine on the table and held his breath at full inspiration for each scan. Scans were taken with a slice width of $10 \mathrm{~mm}$ and an interval of 10 or $15 \mathrm{~mm}$, from the lung apices to the bases. The scan was not gated to lung volume but the radiographer would proceed only if the patient indicated that his lungs were full. At the end of the inspiratory series the sequence was repeated in full, comfortable expiration. The images were then examined for features of bullous lung disease and the volume and vital capacity of any major bulla was calculated as described below.

Two aspects of the interpretation of inspiratory and expiratory computed tomography scans may be worth noting. Firstly, on expiration the lungs shorten, move cranially, and are often scanned with fewer slices than in inspiration. Thus the inspiratory and expiratory scans cannot be matched exactly by slice or bed position alone. To make useful comparable observations some intrapulmonary or bronchial landmark (for example, the carina) must be used to match the slices as closely as possible. Secondly, bullae may change shape during respiration without altering volume, as illustrated in fig 1 . The volume of the bulla in the figure did not alter but its shape changed considerably and assessment of the ventilation of the bulla based on the change of area in a single slice would be inaccurate. To discover whether or not bullae are ventilated therefore their total volume must be measured in inspiration and again in expiration.

In this study the volumes of the bullae were determined by outlining their boundaries in every scan of the inspiratory and expiratory sequence. The area of the bulla contained within each slice was calculated and the sum of the areas was multiplied by the common scan interval. With the early scanner areas were calculated by using the planimeter facility of the hos- pital computer on large size hard copy of the scans. $\stackrel{\vec{F}}{\vec{f}}$ The later scanner was able to calculate the area $\overline{0}$ directly after outlining it with an arbitrary region of $\frac{\mathrm{C}}{\mathrm{O}}$ interest. We tested this method of volume recon- $\frac{\bar{\omega}}{\sigma}$ struction in phantoms of lung like foams over a vol- $\stackrel{\mathbb{Q}}{\circ}$ ume range of $150-12500 \mathrm{ml}$ and found that it was accurate to within $0.2($ SD 68$) \mathrm{ml}(\mathrm{n}=10){ }^{7}$

The assessment of the degree of generalised $\overrightarrow{0}$ emphysema was made empirically according to the $\overrightarrow{\vec{\omega}}$ degree and extent of the destruction of normal lung $\stackrel{\omega}{\omega}$ architecture. There is no generally accepted method $\underset{\vec{x}}{\vec{x}}$ of assessing the degree of pulmonary emphysema seen on computed tomography; although quantification of $\overrightarrow{ \pm}$ the frequency distribution of computed tomography densities appears promising ${ }^{8}$ this was not available to of us. Our assessment included the degree and extent of $\mathrm{F}$ generalised emphysema derived from the appearance of relative transradiancy at the usual window settings, $\vec{z}$ attenuation of blood vessels, and destruction of the parenchymal pattern. These features were noted in the individual case but no attempt was made to relate $\frac{3}{0}$ the apparent degree of emphysema to lung function $\stackrel{\mathbb{Q}}{\longrightarrow}$ or to postoperative improvement.

\section{ROUTINE LUNG FUNCTION TESTS}

Spirometric volumes- $-\mathrm{FEV}_{1}$, forced vital capacity (FVC) - were measured in each patient with a rolling seal spirometer (Ohio 800/810). Lung volumes-total o lung capacity (TLC), residual volume (RV), forced $\stackrel{\circ}{\varnothing}$ respiratory capacity (FRC), and specific airway $\varrho$ conductance (sGaw) - were estimated by whole body $\overrightarrow{\vec{\partial}}$ plethysmography (Fenyves and Gut). All volumes 3 were corrected to BTPS. Single breath carbon monoxide transfer factor (TLCO), 10 second helium alveolar volume (VA) and transfer coefficient $(\mathrm{KCO})$ were

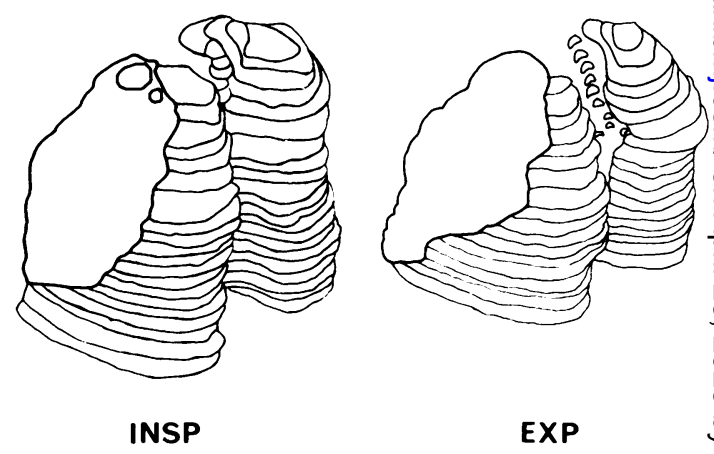

Fig 1 Three dimensional reconstruction (posterior oblique view') of the computed tomography scan of the lungs of a patient with a large bulla in the left upper lobe. The measured 0 volume of the bulla does not change from inspiration to expiration but there is a change in shape due to distortion. This would invalidate the measurement of ventilation from the change in area of the bulla in a single slice. 
estimated with a PK Morgan Respirameter model C. A measure of trapped gas was obtained by subtraction of the alveolar volume from the plethysmographic total lung capacity (TLC-VA). Estimations of blood gas tensions were obtained from 37 patients on the basis of arterialised samples of earlobe blood. Most of the lung function results are presented as percentages of normal values, the regression equations cited by Cotes $^{9}$ being used.

\section{SURGICAL METHODS}

Twelve patients eventually had surgery for their bullae. In 10 patients who had operations performed at the Brompton Hospital, follow up lung function and blood gas measurements were performed between two months and one year after surgery. Two other patients had operations at the referring hospital, which could provide only repeat spirometric values. These two patients and the first three patients operated on locally had full thoracotomies with excision or plication of the bulla. The remainder had a modified Monaldi procedure. In this operation the bulla is approached by a limited thoracotomy and a drain is inserted into the cavity of the bulla, secured by a purse string suture, and left on suction for about seven days. A second drain to protect against pneumothorax is inserted into the pleural space. The operation has previously been considered to be valuable only in patients with a high risk of complication but it is now used for routine cases in this hospital.

\section{Results}

\section{COMPUTED TOMOGRAPHY}

From the appearances of the scan the 43 original patients could be put into two categories. Twenty patients did not have discrete bullae but did have gen-

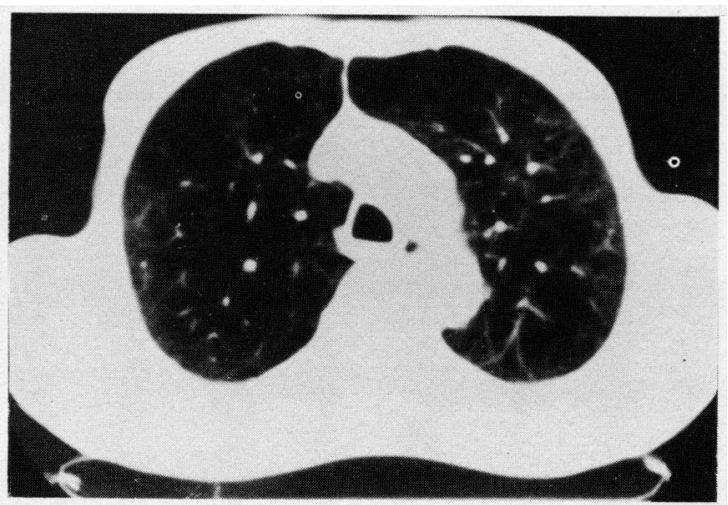

Fig 2 Computed tomography scan from a patient with a suspected bulla in the right lung. Both lungs are emphysematous and the right side is more severely affected; there is no discrete bulla.

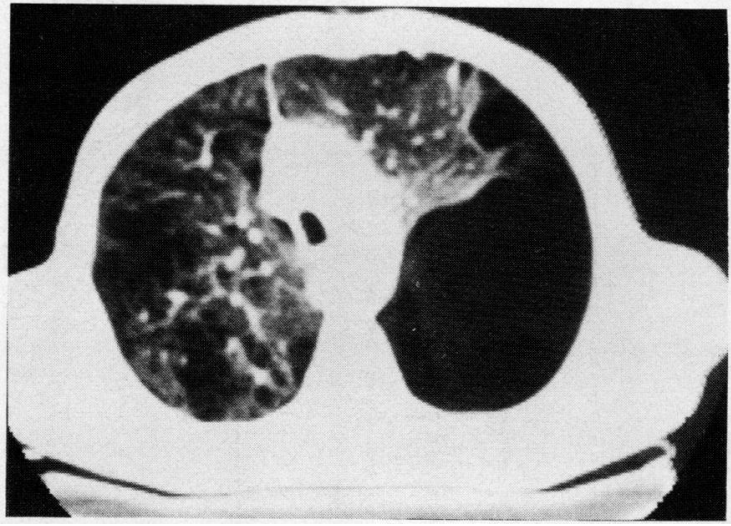

Fig 3 Computed tomography scan from a patient with a well defined bulla in the left upper zone (contrast fig 2). The surrounding lung appears more normal than in the previous figure (different window settings in this example do not allow detailed comparison with fig 2).

eralised emphysema, which was locally severe in the area of the suspected bulla (fig 2). The remaining 23 patients had well defined intrapulmonary bullae with measurable volume. These were surrounded by lung of variable quality (fig 3); in some patients the peribullous lung appeared normal but in most there was emphysema of varying severity.

The volumes and vital capacities of the 23 true discrete bullae are shown in figure 4 . In full inspiration the volumes of the bullae ranged from 158 to $3614 \mathrm{ml}$ (mean $1454 \mathrm{ml}$ ). With few exceptions there was little change in volume at full expiration (mean $1333 \mathrm{ml}$ ). The RV/TLC ratios of most of the bullae therefore were high (89\% (SD 10\%)) and in all cases exceeded those of the surrounding lung. The vital capacity of the bulla was expressed as a proportion of the overall $\mathrm{VC}$ and in 22 patients this was less than $8 \%$. In only one patient did the bulla make a notable contribution to the vital capacity $(440 \mathrm{ml}$ in $2 \cdot 31$-that is, $19 \cdot 2 \%)$.

\section{LUNG FUNCTION}

The results of the routine lung function tests on all 43 patients are shown in table 1. Statistical comparisons (Student's unpaired $t$ test) were made between the results in patients with generalised emphysema and those with discrete bullae. There was no difference between the two groups either in age or in most of the indices of lung function. There were, however, significant differences in TLC, FRC, and Kco. These differences are without practical importance in individuals because the range of values in both groups was wide (fig 5).

SURGER Y

On the basis of the computed tomography appear- 
Bulla Volume

Litres

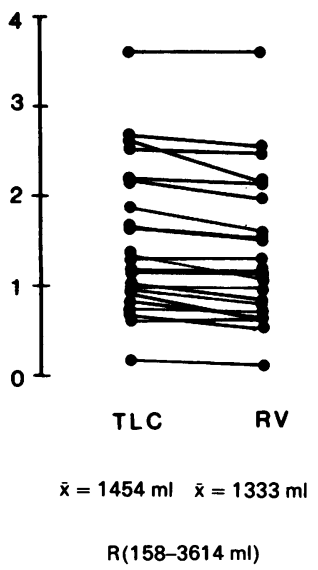

RVITLCX

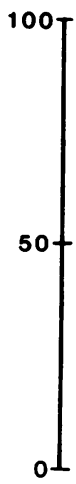

Bulla

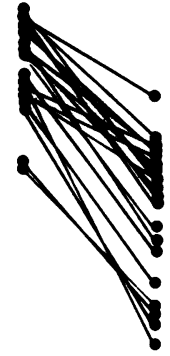

Lung ances, the referring physicians were advised to offer surgery to all those patients with true bullae (except for the patient with the lowest bulla volume). The patients with generalised emphysema were not recommended for surgery. Only 12 patients with true bullae were eventually referred for operation. The decision to withold surgery in the others was made entirely on clinical grounds. There were no major differences in age or lung function between those who underwent

Table 1 Lung function (means with standard deviations in parentheses) in 23 patients with discrete bullae and 20 with locally severe emphysema

\begin{tabular}{|c|c|c|}
\hline & True bullae & Local emphysema \\
\hline $\begin{array}{l}\text { Age } \\
\text { FEV }(\% N) \\
\text { FVC }(\% \mathrm{~N}) \\
\text { TLC }(\% \mathrm{~N}) \\
\text { RV }(\% \mathrm{~N}) \\
\text { FRC }(\% \mathrm{~N}) \\
\text { sGaw }\left(\mathrm{s}^{-1} \mathrm{kPa}^{-1}\right) \\
\text { VA }(\% \mathrm{~N}) \\
\text { TLC }-\mathrm{VA}_{\mathrm{A}}(\mathrm{ml}) \\
\text { TLCO }(\% \mathrm{~N}) \\
\mathrm{KCO}(\% \mathrm{~N}) \\
\mathrm{PaO}_{2}(\mathrm{kPa}) \\
\operatorname{PaCO}_{2}(\mathrm{kPa})\end{array}$ & $\begin{array}{cl}51 \cdot 1 & (10.9) \\
40.3 & (22 \cdot 0) \\
69.2 & (19.6) \\
119.1 & (16.8) \dagger \\
202.2 & (69.5) \\
143 & (36.2)^{*} \\
0.52 & (0 \cdot 6) \\
76.2 & (13.8) \\
3057 & (1586) \\
55.2 & (21.7) \\
78.3 & (26.5)^{*} \\
9.9 & (2 \cdot 1) \ddagger \\
4.9 & (0.6) \ddagger\end{array}$ & $\begin{array}{cl}54.9 & (8.0) \\
32.8 & (20.4) \\
74.0 & (23.8) \\
132.2 & (12.9) \dagger \\
233.6 & (60.4) \\
165.5 & (26.5)^{*} \\
0.40 & (0.6) \\
81.1 & (16.4) \\
3555 & (1083) \\
4.1 & (19.8) \\
59.4 & (28.4)^{*} \\
8.8 & (1.5) \S \\
4.7 & (0.5) \S\end{array}$ \\
\hline
\end{tabular}

* $\mathrm{p}<0.05 ; \mathrm{tp}<0.01 ;$; $\mathrm{n}=20 ; \S \mathrm{n}=17$.

$\% \mathrm{~N}-\%$ predicted normal value; FVC-forced vital capacity; TLC - total lung capacity; RV-residual volume; FRC - functional residual capacity; SGaw-specific airways conductance; VA-alveolar volume; TLCo-transfer factor; Kco-transfer coefficient; $\mathrm{PaO}_{2}$-arterial oxygen tension; $\mathrm{PaCO}_{2}$-arterial carbon dioxide tension.

Conversion: SI to traditional units-sGaw: $1 \mathrm{~s} \mathrm{~s}^{-1} \mathrm{kPa}^{-1}=10 \mathrm{~s}^{-1} \mathrm{~cm}$ $\mathrm{H}_{2} \mathrm{O}^{-1}$; blood gas tensions: $1 \mathrm{kPa}=7.5 \mathrm{~mm} \mathrm{Hg}$.
vC Bulla/vC Lung

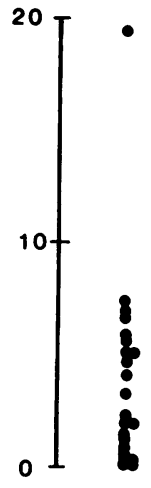

$\overline{\mathrm{x}}=\mathbf{4 . 0 \%}$

$R(0-19.2 \%)$
Fig 4 Bulla volume and ventilation. Left: Volumes of bullae in 23 patients in inspiration and expiration. ஸे Middle: ( $R V / T L C)$ residual volume/total lung capacity ratios of the bullae always exceed those for the lung as a whole. Right: Vital capacity (VC) of bulla/VC lung ratios: in a vital capacity breath most bullae did not contribute to ventilation, only one bulla contributing as much as $19 \cdot 2 \%$.

surgery and those who did not, but the bullae in the surgical group were larger (table 2). All patients who went for surgery survived the operation and reportecb an improvement in their quality of life, though nos objective estimates of exercise tolerance wereo obtained.

The postoperative lung function results are shown in table 3, and graphical examples of the changes in $\vec{b}$ $\mathrm{FEV}_{1}, \mathrm{FVC}_{\text {, TLCO, KCO, and arterial oxygen tension }}$

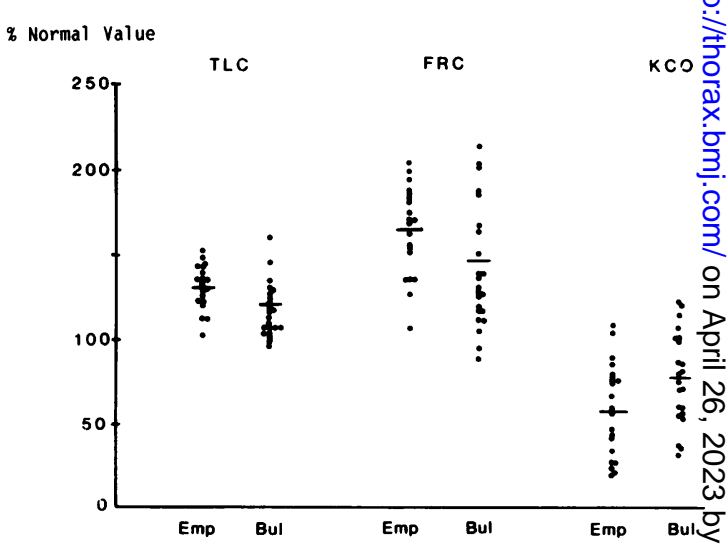

Fig 5 Significant differences in lung function between those patients with true bullae (Bul) and those with generalised emphysema (Emp). The differences in total lung capacity $(T L C)$, functional residual capacity (FRC), and transfer coefficient $(\mathrm{KCO})$ are not of practical importance because of the wide range. 
Table 2 Lung function (means with standard deviations in parentheses) in 12 patients treated surgically and 11 patients with discrete bullae not submitted to surgery

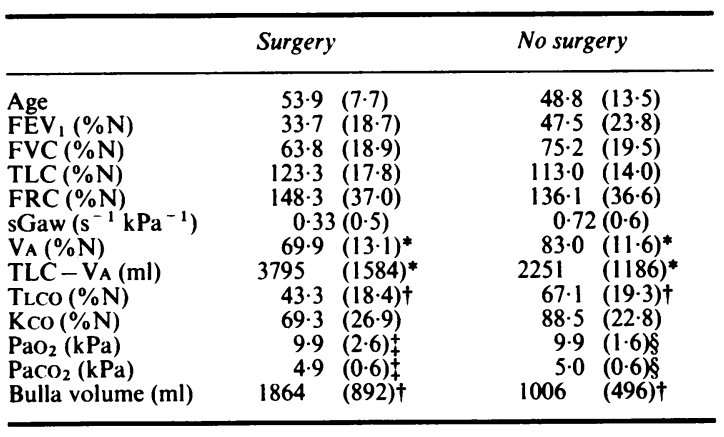

${ }^{*} \mathrm{p}<0.05 ; \mathrm{tp}<0.01 ;$ tn $=11 ; \S \mathrm{n}=9$

Abbreviations and units as in table 1 .

$\left(\mathrm{PaO}_{2}\right)$ in figure 6. Statistical comparison between the preoperative and postoperative values was made by Student's $t$ test. There were significant improvements in the mean values of $\mathrm{FEV}_{1}(16 \%)$, FVC $(21 \%)$, sGaw $\left(0.5 \mathrm{kPa} \mathrm{s}^{-1}\right)$, and $\mathrm{VA}_{\mathrm{A}}(10 \%)$. There were also significant decreases in the mean values of lung volumes TLC $(13 \%)$, FRC $(23 \%)$, RV $(71 \%)$, and gas trapping TLC $-V_{A}(1421 \mathrm{ml})$. The improvements in spirometric values were less impressive in those patients with poor function before surgery but the reductions in lung volumes were more consistent. There were no significant changes in carbon monoxide transfer or blood gas tensions, and these values in the figure are clustered around the line of identity.

There were general relationships between the increases in FEV 1 and VC, reduction in lung volumes, and the bulla volume (fig 7). The improvements in these values was less than the bulla volume in most cases and there were no strong correlations between them (best $r$ value 0.38 ). Two patients actually showed reductions in lung volumes that were larger than the bulla volumes. One patient had a small increase in TLC but this was offset by an improvement of 2 litres in VC at the expense of RV. There was also no indication that those who underwent the Monaldi operation had any poorer results than those who had excision or plication. The numbers are, however, too small for statistical comparison.

\section{Discussion}

It is now well recognised that computed tomography can demonstrate the distribution of bullous emphysema, in particular disclosing disease that is invisible on the plain radiograph or obscured by deformities of the rib cage. Surprisingly, however, several of the patients with radiographic appearances suggesting bullae did not have true discrete bullae confirmed by computed tomography.

Bullae are thought to cause symptoms by occupying space, by acting as dead space, or by compressing healthy lung. The relative importance of these factors will vary from case to case but previous reports have emphasised the demonstration of dead space ventilation or compression as preconditions for surgery. The results of our measurements of bulla volume confirm the findings of Pride and others ${ }^{2}$ that most bullae do not change their volume during a breath. Where there is a change in volume a proportion of the change may be explained by gas compression and expansion. Since the scans were taken in relaxed inspiration and expiration, however, this is unlikely to account for much of the difference.

Table 3 Preoperative and postoperative lung function in 10 patients

\begin{tabular}{|c|c|c|c|c|}
\hline & \multicolumn{2}{|l|}{ Preoperative } & \multicolumn{2}{|l|}{ Postoperative } \\
\hline & $\operatorname{Mean}(S D)$ & Range & Mean (SD) & Range \\
\hline 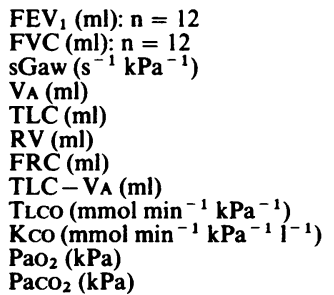 & $\begin{array}{l}1014(494) \\
2630(806) \\
0.38(0.45) \\
3986(804) \\
7646(1203) \\
4888(1582) \\
6181(1569 \\
3660(1424) \\
3.78(1.52) \\
0.97(0.43) \\
9.91(2.60) \\
4.89(0.64)\end{array}$ & $\begin{array}{l}350-2130 \\
1410-4510 \\
0 \cdot 06-1 \cdot 39 \\
2760-5480 \\
5570-10000 \\
2180-8100 \\
4230-9450 \\
1680-6940 \\
1 \cdot 59-6 \cdot 23 \\
0.43-1.93 \\
5 \cdot 9-140 \\
3.6-5 \cdot 9\end{array}$ & $\begin{array}{l}1533(698) \dagger \\
3503(860) \ddagger \\
0.86(0.9)^{*} \\
4292(925)^{*} \\
6953(1353)^{*} \\
3375(1074) \dagger \\
5053(1346) \dagger \\
2267(1042) \dagger \\
4.05(1.64) \\
0.92(0.39) \\
9.82(2.04) \\
4.92(0.79)\end{array}$ & $\begin{array}{l}460-2660 \\
2120-4930 \\
0 \cdot 16-2 \cdot 57 \\
3270-6680 \\
4960-9760 \\
1370-4760 \\
3030-7460 \\
630-3820 \\
1 \cdot 60-7 \cdot 42 \\
0 \cdot 37-1 \cdot 67 \\
6 \cdot 7-13 \cdot 0 \\
3 \cdot 6-6 \cdot 3\end{array}$ \\
\hline
\end{tabular}

$* \mathrm{p}<0.05 ;$ tp $<0.01 ; \neq \mathrm{p}<0.001$.

Abbreviations as in table 1 .

Conversion: SI to traditional units-TLCo and KCo: multiply by 2.99 . 

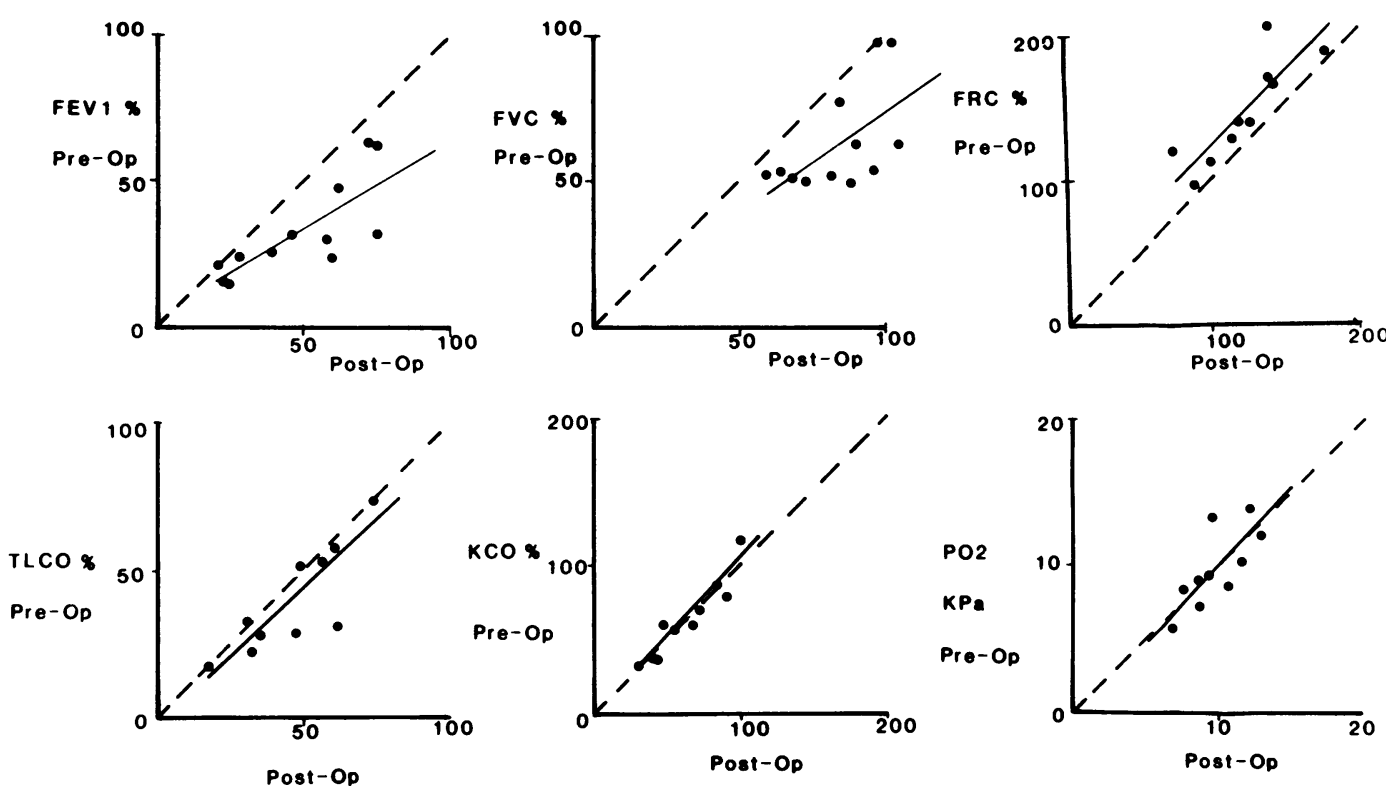

Fig 6 Examples of preoperative and postoperative function, expressed as $\%$ predicted, except for arterial oxygen tension $\left(\mathrm{PaO}_{2}\right)$. The improvements in spirometric values were least impressive in those patients with poor preoperative values. The reduction in lung volumes - for example, functional residual capacity (FRC)-were more consistent. There were no significant improvements in transfer factor $(\mathrm{TLCO})$, transfer coefficient $(\mathrm{KCO})$, or $\mathrm{PaO}_{2}$. The solid lines are regression lines and the dotted lines those of identity.

Change in bulla volume accounted for a significant proportion of the vital capacity in only one case. In most patients therefore, additional dead space ventilation was small and the bullae did not ventilate preferentially. It is important to note that even if bullae have significant vital capacities demonstrable under scanning conditions, they may have long time constants of filling or emptying and so contribute little to steady state tidal ventilation.

All the patients with true bullae who underwent surgery survived and reported improvements in their symptoms. This was particularly encouraging since

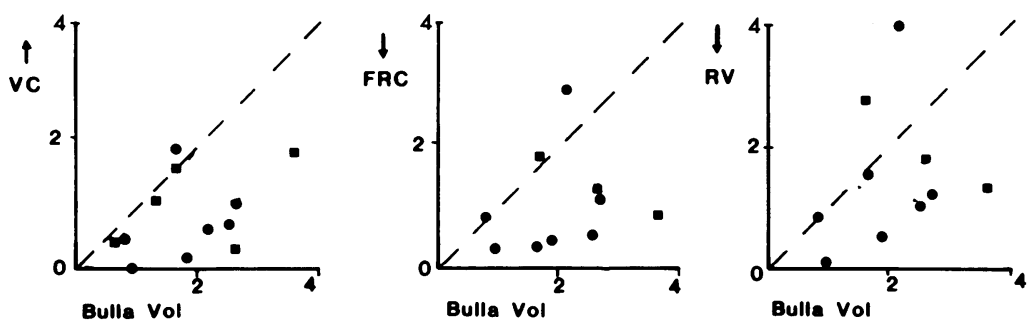

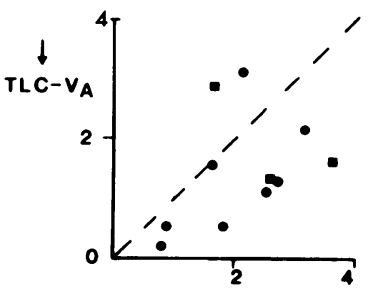

Bulla Vol

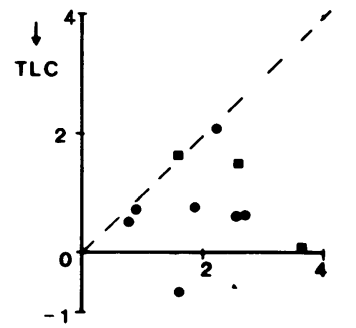

Bulla Vol
Fig 7 Postoperative increase in vital capacity $(V C)$ and reduction in lungo volumes in relation to the original bulla volume (all $\mathrm{N}$ volumes in litres). The relationship shows poor correlation, which may be $\mathrm{\omega}$ partly attributable to failure of the operation to ablate the bulla completely. The Monaldi procedure seems worse in this respect. 
two thirds of the patients had an $\mathrm{FEV}_{1}$ less than 1 litre, although none of them had excessive sputum production or hypercapnia, which are also considered to be indicators of poor prognosis. The postoperative improvements in objective tests of lung function included reductions in static lung volumes and increases in $\mathrm{FEV}_{1}$ and vital capacity. The absence of improvement in gas transfer and blood gas tensions argues against the release of lung compression as the only mechanism of symptom relief. This is contrary to the findings of Pride et al and the discrepancy is difficult to explain, except perhaps that the bullae in their series are likely to have been larger and easily demonstrable on plain radiograph, and that their removal allowed more lung to expand. In our series the absence of dead space ventilation and the failure to obtain improvement in gas exchange suggest that reduction in lung volume alone is likely to be the most important factor in improvement; such a reduction would result in decrease of the elastic work of breathing and would return the respiratory muscles to a more efficient configuration. In addition, the sensation of discomfort perceived via chest wall receptors may be reduced. The reduction in lung volume occurred consistently in all patients and is presumably related directly to the obliteration of the space. Most operations, however, appear not to ablate the space completely, and indeed small pockets of bulla were visible in some patients who had postoperative computed tomography scans. This is likely to be one reason for the poor relationships between the bulla volume and the postoperative reduction in lung volumes.

The ability of various techniques to assess the quality of lung surrounding a bulla depends on whether the bulla contributes to ventilation. When bullae do not change volume a functional description of the non-bullous lung is obtained from overall lung function tests. If, however, bullae do ventilate significantly, then the quality of surrounding lung will need to be assessed by other techniques. Unfortunately both the available methods for assessing regional lung function, using either radioisotopes or endobronchial sampling, have disadvantages. Endobronchial tests, such as the single breath argonfreon test, cannot provide an accurate quantitative estimate of function in the presence of airway obstruction. ${ }^{10}$ Radioisotope ventilation and perfusion scans can fractionate function between whole lungs but they cannot with any confidence be used to assess function of the lung surrounding a bulla since, as with the plain radiograph, lung and bulla will be superimposed. A potentially good assessment of surrounding lung can be obtained by computed tomography, when the presence of emphysema can be detected and distinguished from normal lung, but at present this description is qualitative and further study is needed to identify the effects of bullae on adjacent lung.

In the patients reported here there appeared to be no disadvantage to those who underwent the simpler surgical procedure. In practice the Monaldi technique does have several theoretical advantages. The incision is small and can be made directly over the bulla with computed tomography guidance. There is no danger of removing potentially functioning lung as there is with excision or lobectomy, but it may incompletely deflate some bullae and later require a second procedure. Finally, since the operation is easier and shorter there may be less risk of postoperative complications in the compromised patient.

In summary, computed tomography has contributed to the preoperative assessment of patients with bullous lung disease and poor overall lung function. It can identify bullae that are potentially operable and measure their volume and ventilation, thus improving the sensitivity of whole lung function tests. The surgical removal of moderate sized bullae, even where the $\mathrm{FEV}_{1}$ is less than 1 litre and the surrounding lung is emphysematous, has a low mortality rate and is associated with a reduction of lung volume and the relief of symptoms. A reduction in dead space ventilation or release of lung compression may be relatively unimportant mechanisms of the symptom relief that follows operation for smaller bullae. For practical purposes therefore it may not be necessary to demonstrate that either lung compression or dead space ventilation is present to justify surgery. Merely demonstrating by computed tomography that an appreciable space exists may be sufficient grounds for expecting improvement after operation.

Our grateful thanks go to the radiographers of Westminster and Bromptom Hospitals, particularly to Wendy Jordan. Most of the operations were performed by $\mathrm{Mr}$ Peter Goldstraw, who has also given invaluable advice. The manuscript was typed by Julie Anagnostou.

\section{References}

1 Pearson MG, Ogilvie C. Surgical treatment of emphysematous bullae: late outcome. Thorax 1983; 38:134-7.

2 Pride NB, Barter CE, Hugh-Jones P. The ventilation of bullae and the effect of their removal on thoracic gas volumes and tests of overall pulmonary function. Am Rev Respir Dis 1973;107:83-98.

3 Potgieter PD, Benetar SR, Hewitson RP, Ferguson AD. Surgical treatment of bullous lung disease. Thorax 1981;36:885-90.

4 Morgan MDL, Strickland B. Computed tomography in the assessment of bullous lung disease. $\mathrm{Br} J$ Dis Chest 
1984;78:10-25.

5 Carr DH, Pride NB. Computed tomography in preoperative assessment of bullous emphysema. Clin Radiol 1984;35:43-5.

6 Fiore D, Biondetti PR, Sartori F, Calabro F. The role of computed tomography in the evaluation of bullous lung disease. J Comp Asst Tomog 1982;6(1):105-8.

7 Denison DM, Morgan MDL. CT estimates of gas and tissue volumes of the lung [Abstract]. Clin Sci 1985;6(11):30P.
8 Hayhurst MD, Flenley DC, McLean A, Wightman AJA, MacNee W, Wright D, Best J. Diagnosis of pulmonary emphysema by Computerised Tomography. Lancet 1984;ii:320-2.

9 Cotes JE. Lung function: principles and application in medicine. 4th ed. Oxford: Blackwell Scientific Publica- $\triangle$ tions, 1979.

10 Denison DM, Waller JF. Interpreting the results of regional single-breath studies from the patient's point $\overrightarrow{0}$ of view. Clin Respir Physiol 1982;18:339-51. 